\title{
Multiport resonant DC-DC converter
}

Y. K. Tran, D. Dujić, and P. Barrade

This material is posted here with permission of the IEEE. Such permission of the IEEE does not in any way imply IEEE endorsement of any of EPFL's products or services. Internal or personal use of this material is permitted. However, permission to reprint / republish this material for advertising or promotional purposes or for creating new collective works for resale or redistribution must be obtained from the IEEE by writing to pubs-permissions@ieee. org. By choosing to view this document, you agree to all provisions of the copyright laws protecting it. 


\section{Multiport Resonant DC-DC Converter}

\author{
Yan-Kim Tran, Dražen Dujić \\ École Polytechnique Fédérale de Lausanne - EPFL \\ Power Electronics Laboratory - PEL \\ Station 11, CH-1015, Lausanne \\ yan-kim.tran@epfl.ch,drazen.dujic@epfl.ch
}

\author{
Philippe Barrade \\ HES-SO Valais-Wallis \\ Institute of System Engineering \\ PO box 2134, CH-1950, Sion \\ philippe.barrade@hevs.ch
}

\begin{abstract}
This paper presents a multiport galvanically isolated LLC resonant DC-DC converter suitable for DC applications. A three-port structure is analyzed, with full bidirectional power flow capabilities, simple control and behavior similar to that expected from a DC transformer. Each port is equipped with half-bridge modules accompanied with tuned resonant tank, partly realized with elements of a multiwinding high frequency transformer. With some restrictions that are explained in the paper, each port can be a sink or a source of power, depending on the presence of switching action on the half-bridge stage. To maximize the efficiency of the system, no active control is applied and the system operates at fixed switching frequency, below the resonant frequency of the system.
\end{abstract}

Keywords-DC-DC; resonant conversion; multiport converter.

\section{INTRODUCTION}

Future of electrical energy production and distribution is oriented towards the use of distributed energy resources interconnected with existing AC grids, or emerging DC grids. Increased volume of PV installations on small systems, such as private buildings, allows to private entities to participate in evolution of energy sector and to become consumer and producer at the same time. The proximity of generation and consumption sites has a positive effect on the system efficiency and avoids long distance power transfer and multiple conversion stages. These micro-grids composed of multiple participants in energy exchange, may suffer from the instabilities caused by the volatile nature of renewable sources, such as sudden and temporary shading on PV installations. This can be in part compensated through interconnections between multiple micro-grids or through inclusion of storage elements [1], such as ultra-capacitors or batteries, as supporting measures to deal with rapid fluctuations of power supply and demand.

Considering that PV generation as well as electrical storage technologies mentioned earlier are naturally DC, there is an increased interest in development of DC grids and enabling power electronic technologies. Flexibility and simplicity of interconnection technologies as well as suitable protection technologies are the key to enable new grids. In that frame, multi-port power electronic converters could be a solution to integrate different storage technologies into micro-grids, while providing fully bidirectional and reliable power flows. This paper, proposes such a multi-port DC-DC converter, characterized by galvanic coupling between different ports of different voltage/power ratings, bidirectional power flow and simple operating principles.

So far, only a few investigations related to high power multiport converters have been reported. Work of [2] deals with converters based on interconnection through a common DC bus. Although there are clearly some advantages due to the simplicity of the control, voltage range is limited and defined by choice of the bus voltage. This limitation can be overcome by means of electromagnetic coupling through medium or high frequency transformers. In [3],[4] and [5] authors have presented solutions derived from the Dual Active Bridge (DAB) or Dual Half Bridges (DHB) with inclusion of additional ports. Some of the ports are operated using full bridges and duty cycle control, while the others are operated with half bridges in combination with phase-shift control. Limited soft switching operating range of $\mathrm{DAB}$, resulted in considerations towards the resonant converters thanks to their advantages in reduced switching losses. Multi-port LLC converter has been presented in [6] and consists of a three winding medium frequency transformer (MFT) with distributed resonant capacitors tuned to multiple leakage inductors, in order to create resonant tank. Each electrical port can be either configured as halfbridge or full-bridge, and all ports are switched at resonant frequency with the same phase and $50 \%$ duty cycle. Authors have presented configuration where one port act as a source, while two other ports are acting as sink (load). Experiments from a low power setup (300W), demonstrate good load regulation. On the other hand, work of [7] shows a similar topology where two ports are configured as sources and are equipped with resonant capacitors, while the third port has only a rectifier as it is behaving as the load. Authors have demonstrated controllable power flow and sharing between sources, through a phase shift control. While, numerous works have been dealing already with LLC tank design [8] and [9], this is not the case when it comes to the tank design of a multi-port LLC converter.

This paper proposes a topology based on a multi-winding medium frequency transformer as shown in Fig. 1. Proposed topology is fully bidirectional, even when one of the three ports does not participate in energy exchange. For this reason, the resonant tank is not located on a single port, like in [6], but is split and distributed between all the three ports. To keep the number of semiconductor devices at minimum, all switching cells are in half-bridge configuration and use split DC bus. 


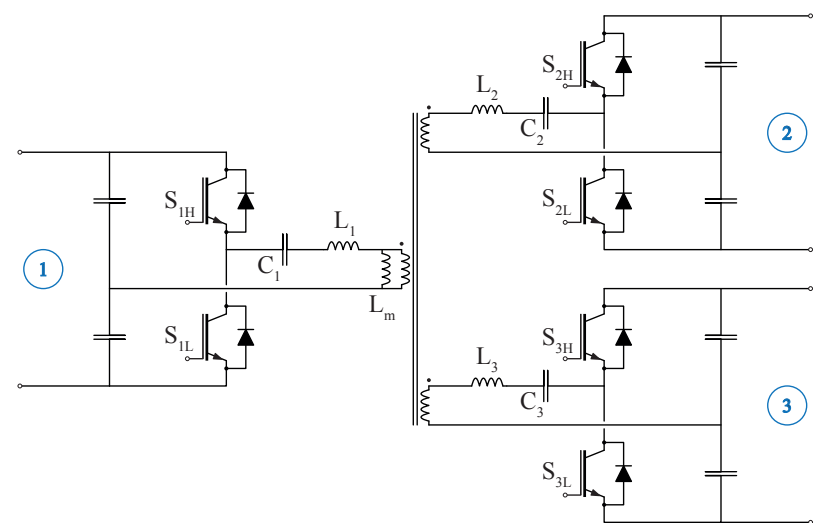

Fig. 1. Proposed topology of multi-port resonant DC-DC converter, based on multi-winding medium frequency transformer, distributed resonant tank and half-bridge power stages.

In general, electric ports may have different power ratings $\left(P_{1_{\max }} \neq P_{2_{\max }} \neq P_{3_{\max }}\right)$ and different rated operating voltages $\left(V_{D C_{1}} \neq V_{D C_{2}} \neq V_{D C_{3}}\right)$. These are adjusted through MFT turn ratios $n_{1}, n_{2}$, and $n_{3}$, which are also different, in general. Each resonant tank is realized as pair of $\left(L_{i} ; C_{i}\right)$, and is sized accordingly, considering certain restrictions related to $V_{i}$ and $P_{\max _{i}}$, as discussed in the paper. In principle, $L_{i}$ is realized as the leakage inductance of the winding $i$ with, eventually, an additional inductor. The proposed converter is intended to operate as a simple DC transformer without any complex control scheme, and only the ports that are sourcing the power are actively switched. Ports that are power sink in a given point in time, are not switched and are acting as simple diode rectifier (voltage-doubler effectively, due to chosen connection). In order to maintain zero voltage switching (ZVS)of the switched port and zero current switching (ZCS) of passive rectifiers, the proposed converter is operated near and below resonant frequency $\left(f_{s w}<f_{0}\right)$.

This paper is organized as follows: In Section 2 the main modes of operation of multiport resonant DC-DC converter are described. Resonant tank design is presented in Section 3 , while the characteristics simulation results are presented in section 4. Summary and conclusion are provided in Section 5.

\section{Proposed Operating Modes}

Treating all electrical ports as identical, two main operation modes of the proposed converter configuration can be distinguished. In the mode $\mathrm{A}$, one of the three port is acting as a source and is transferring power to the two other ports, that act as loads (power sink). On the other hand, in the mode B, two ports out of three are sources and supply power to the third port. For a time being, it is assumed that ratings of each port can be freely selected, while in reality this may not be the case and certain limitations will be present due to application requirements and due to limits of power semiconductor devices, as discussed later. Both operating modes are illustrated in Fig. 2 and Fig. 3, respectively.

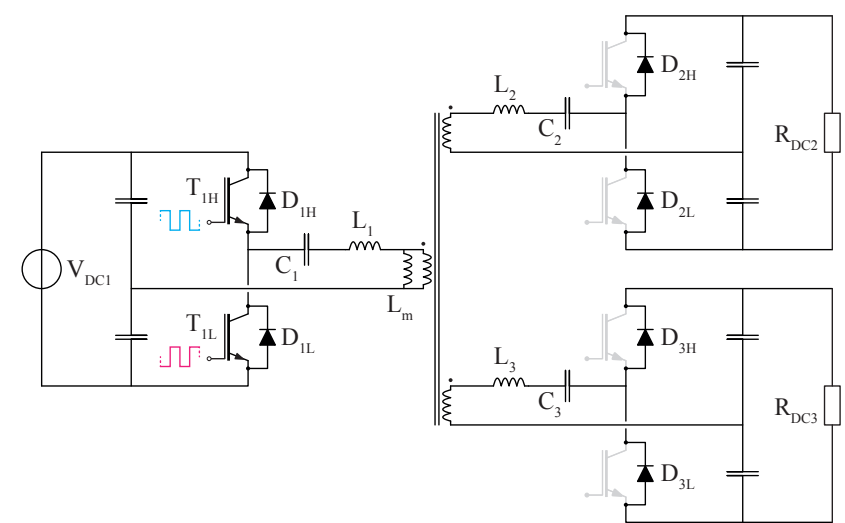

Fig. 2. Mode A with power transfer from the port 1 to the ports 2 and 3 .

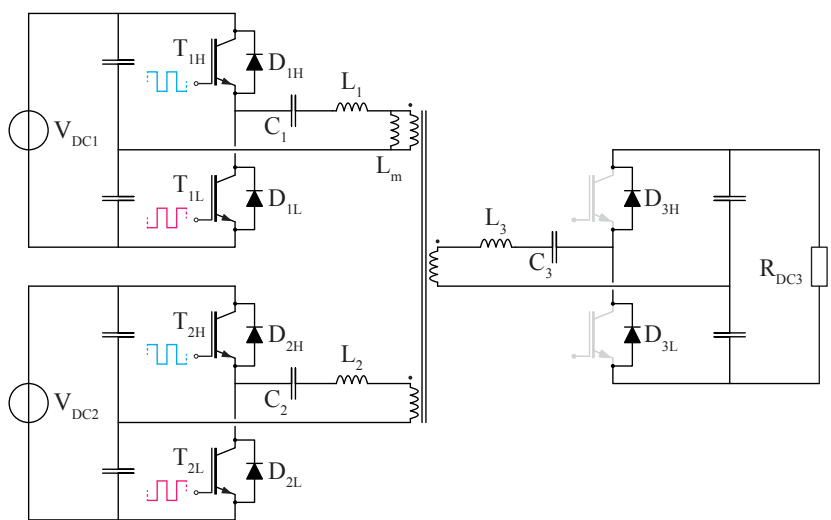

Fig. 3. Mode B with power transfer from the ports 1 and 2 to the port 3 .

As with simple LLC resonant converter, target is to maintain ZVS conditions during turn-on for the switched devices, as well as ZCS for diode rectifiers.

\section{A. Mode A}

To describe mode A operation, it is assumed that port 1 is source feeding ports 2 and 3, as shown in Fig. 2. For that reason, semiconductor devices of port $1\left(T_{1 H}, T_{1 L}\right)$ are switched, while active semiconductor devices on the ports 2 and 3 are turned off and only the free-wheeling diodes $\left(D_{2 H}, D_{2 L}, D_{3 H}, D_{3 L}\right)$ are used for passive rectification. This simplifies the control, which only has to recognize change in power flows and introduce or suppress switching actions. Switches $T_{1 H}$ and $T_{1 L}$ are operated at fixed switching frequency $f_{s w}=f_{n} f_{0}$ with $f_{n}<1$ and a constant duty cycle of approximately $50 \%$ (considering dead-time). This mode of operation is easy to achieve, without any special efforts.

\section{B. Mode B}

It is considered that port 1 and 2 are supplying the power to the port 3. To achieve power transfer, semiconductor devices of port $1\left(T_{1 H}, T_{1 L}\right)$ and that of port $2\left(T_{2 H}, T_{2 L}\right)$ are switched synchronously with the same switching frequency. 
Active devices on the port 3 are turned off and only passive rectification is used to supply the load. To achieve operation in this mode, resonant tank design must be done correctly.

\section{Multiport Resonant TAnk Design}

The design of the resonant tank requires proper sizing of all distributed $L_{i}$ and $C_{i}$ in a way that guaranties soft switching conditions for any operating point within the power specification given by $P_{i_{\max }}$ (per port). As the converter is operated in the vicinity of resonant frequency $f_{0}$, first harmonic approximation (FHA) is considered accurate enough and is used during the design.

\section{A. Single resonant tank design}

The voltages generated by the half-bridge stages, namely square-waves of frequency $f_{s w}$ and magnitude $V_{D C_{i}} / 2$ are approximated by their first harmonic. The resonant currents $\left(I_{A C_{i}}\right)$ are estimated from their DC values $\left(I_{D C_{i}}\right)$.

$$
\begin{aligned}
V_{A C}(t) & =\frac{2}{\pi} V_{D C} \sin \left(2 \pi f_{s w} t+\varphi_{v}\right) \\
I_{A C}(t) & =\pi I_{D C} \sin \left(2 \pi f_{s w} t+\varphi_{i}\right)
\end{aligned}
$$

The effective DC loads are modeled as resistors, and are referred through a rectifiers to equivalent $\mathrm{AC}$ loads.

$$
R_{D C}=\frac{V_{D C}^{2}}{P} ; \quad R_{A C}=\frac{2}{\pi^{2}} R_{D C} ; \quad R_{A C_{\min }}=\frac{2 V_{D C}^{2}}{\pi^{2} P_{\max }}
$$

Note that $R_{A C}$ may vary between $\infty$ and $R_{A C_{m i n}}$ which corresponds to an operating point between 0 and $P_{\max }$.

The behavior of the resonant tank (see figure 4), can be described using the quality factor $Q$ defined as ratio of the tank impedance $Z_{r}$ and the effective AC load $R_{A C}$.

$$
Q=\frac{Z_{r}}{R_{A C}} \quad Z_{r}=\sqrt{\frac{L_{r}}{C_{r}}}
$$

Considering normalized DC transfer function of the resonant tank (see Fig. 5) for a given $Q$, the preferred operating region with ZVS behavior is in the inductive part of resonant tank characteristics. In terms of frequency, it corresponds to the frequencies higher than the frequency of the DC gain peak of this $Q$. Note that it is assumed that $Z_{r}$ is known, and therefore $Q$ changes with a change of a load. Since the peak gain is decreasing and moving to the right with increasing $Q$, there is a limited operational range due to fixed switching frequency, that has to be considered. For a selected switching frequency, $Q$ can take values between 0 and $Q_{\text {limit }}$, where peak gain is exactly at switching frequency for $Q_{\text {limit }}$. Therefore, with some margin $Q$ must be chosen small enough (at least smaller than $Q_{\text {limit }}$ ) in order to size $L_{r}$ and $C_{r}$ in a way to preserve tank inductive behaviour and provide soft switching conditions.

Evaluation of the allowed range of $Q$ has been already subject of several publications, and authors of [8] use the criteria:

$$
Q \leq \frac{1}{\sqrt{L_{m} / L_{r}}}
$$

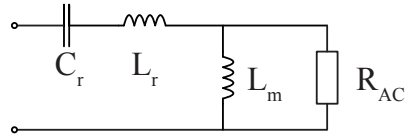

Fig. 4. Simplified resonant tank with equivalent AC load.

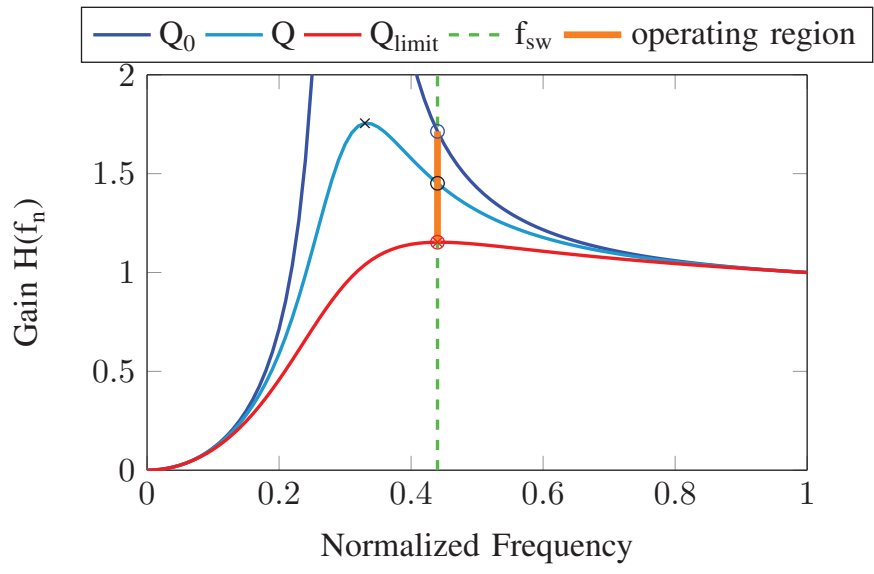

Fig. 5. DC gain for no load conditions $\left(Q_{0}\right)$ and maximal allowed load conditions $\left(Q_{\text {limit }}\right)$. For a given switching frequency, the behavior of the tank is inductive for the $Q$ between $Q_{0}$ and $Q_{\text {limit }}$.

with $Q_{\text {crit }}$ being the maximal value of $Q$ that fulfills this condition. Up to this point, $L_{r}$ is not defined, but through a few substitutions we can extract a $Q_{\text {crit }}$ depending on $R_{A C}$ and $L_{m}$

$$
L_{r}=\frac{Z_{r}}{\omega_{0}} \quad Z_{r}=Q_{c r i t} R_{A C_{m i n}} \quad Q_{c r i t}=\sqrt{\frac{L_{r}}{L_{m}}}
$$

resulting in:

$$
Q_{c r i t}=\frac{R_{A C_{m i n}}}{\omega_{0} L_{m}}
$$

\section{B. Distributed resonant tank design}

Due to bidirectional power flow, the proposed topology does not use single resonant tank located on only one port. This resonant tank could be designed using criteria from previous sub-section. Instead, the resonant tank is distributed among multiple ports, and the aforementioned criteria cannot be used as it is. The approach followed during the design considers three resulting resonant tanks seen from the three different ports, which must all satisfy the Q-criteria for three different $R_{A C_{m i n}}$, respectively. In this way, a $Q_{c i_{i}}$ can be evaluated for each port considering $R_{A C_{1,2,3}}$ and $L_{m_{1,2,3}}$. Since $L_{m}$ is allocated to a the reference port (number 1 in this example), the magnetizing inductance for the other ports are given by:

$$
L_{m_{1}}=L_{m} \quad L_{m_{2}}=L_{m} \frac{n_{2}^{2}}{n_{1}^{2}} \quad L_{m_{3}}=L_{m} \frac{n_{3}^{2}}{n_{1}^{2}}
$$

The quality factors for each port are then obtained combining the equation (3) and (8) into (7). The equivalent impedance 
$Z_{\text {equ }}$ seen from the port $i(i=1,2,3)$. According to the quality factor criteria, it must satisfy the condition:

$$
Z_{\text {equi }} \leq Q_{\text {crit }_{i}} R_{A C_{i}}
$$

The resonant frequency must be the same for all the three ports, due to the synchronized switching. The common resonant frequency $\omega_{0}$ is used to define the resonant capacitor of each sub-tank from its resonant inductor:

$$
C_{i}=\frac{1}{\omega_{i}^{2} L_{i}} \quad \omega_{1}=\omega_{2}=\omega_{3}=\omega_{0}
$$

Each sub-tank description can be then limited to:

$$
Z_{i}=\omega_{0} L_{i}
$$

The equivalent tank impedance seen from port $i$ can be evaluated by the sum of the sub-tank $Z_{i}$ and the parallel combination of the two remaining tanks ( $j$ and $k$ ), taking into account the transformer turn ratios:

$$
Z_{e q u_{i}}=\omega_{0}\left[L_{i}+\left(\frac{1}{\left(n_{i} / n_{j}\right)^{2} L_{j}}+\frac{1}{\left(n_{i} / n_{k}\right)^{2} L_{k}}\right)^{-1}\right]
$$

Combining (9) and (12) and solving the following system gives a set of $L_{i}$ that can be used to properly distribute elements of the resonant tank between ports.

$$
\left\{\begin{array}{l}
L_{1} \leq \frac{Q_{\text {crit }_{1}} R_{A C_{1}}}{\omega_{0}}-\left(\frac{1}{\left(n_{1} / n_{2}\right)^{2} L_{2}}+\frac{1}{\left(n_{1} / n_{3}\right)^{2} L_{3}}\right)^{-1} \\
L_{2} \leq \frac{Q_{c r i t_{2}} R_{A C_{2}}}{\omega_{0}}-\left(\frac{1}{\left(n_{2} / n_{1}\right)^{2} L_{1}}+\frac{1}{\left(n_{2} / n_{3}\right)^{2} L_{3}}\right)^{-1} \\
L_{3} \leq \frac{Q_{c r i t_{3}} R_{A C_{3}}}{\omega_{0}}-\left(\frac{1}{\left(n_{3} / n_{1}\right)^{2} L_{1}}+\frac{1}{\left(n_{3} / n_{2}\right)^{2} L_{2}}\right)^{-1}
\end{array}\right.
$$

Note that the power sharing between feeding ports, in mode $\mathrm{B}$, can be approximated by the ratio of the distributed inductors taking in account the turn ratios of the transformer:

$$
\frac{P_{i}}{P_{j}}=\frac{n_{i}^{2}}{n_{j}^{2}} \frac{L_{j}}{L_{i}}
$$

\section{Simulation Results}

To verify sizing of the distributed resonant tank as well as different modes of operation, simulation of the proposed topology have been carried out in PLECS. Arbitrary voltage and power ratings are chosen for all the three ports. Nevertheless, care is taken that ratings make sense in terms of possible application, where port 1 is sized in a way to meet demands of lower rated ports 2 and 3 . In addition, port 3 ratings are made much smaller, assuming some auxiliary functions (energy storage) of lower rated power. The ratings chosen for simulation are shown in Table I.

The magnetizing inductance (seen from reference port 1) is designed as $L_{m}=3 \mathrm{mH}$ in order to limit the magnetizing current to $40 A_{p k-p k}$. This is also turn-off current for switches of port 1, and is largely load independent. The resonant frequency is selected slightly above the switching frequency $F_{s w}=5 \mathrm{kHz}\left(f_{n}=0.95\right)$. Distributed resonant tank is sized
TABLE I

RATINGS USED FOR SIMULATIONS

\begin{tabular}{|l|l|l|}
\hline Port & Voltage & Power \\
\hline 1 & $3600[\mathrm{~V}]$ & $250[\mathrm{~kW}]$ \\
\hline 2 & $1500[\mathrm{~V}]$ & $200[\mathrm{~kW}]$ \\
\hline 3 & $750[\mathrm{~V}]$ & $50[\mathrm{~kW}]$ \\
\hline
\end{tabular}

using the method described in section III-B and according to the ratings specified in table I.

The results for tank components are shown in table II.

TABLE II

DISTRIBUTED RESONANT TANK PARAMETERS

\begin{tabular}{|l|l|l|l|}
\hline Inductor & Value & Capacitor & Value \\
\hline$L_{1}$ & $12.0 \mu \mathrm{H}$ & $C_{1}$ & $68.2 \mu \mathrm{F}$ \\
\hline$L_{2}$ & $3.1 \mu \mathrm{H}$ & $C_{2}$ & $261.8 \mu \mathrm{F}$ \\
\hline$L_{3}$ & $13.1 \mu \mathrm{H}$ & $C_{3}$ & $62.8 \mu \mathrm{F}$ \\
\hline
\end{tabular}

Both modes of operation (A and B) are simulated considering different loading conditions (different values of $R_{D C}$ ) to demonstrate the behavior of the converter over the full operational range.

\section{A. Operation in the Mode A}

In this mode, port 1 is actively switched while port 2 and 3 are feeding passive loads connected through rectifiers. Three operating points are shown in Figs. 6, 7 and 8 where the loading conditions are adjusted by changing the values of $R_{D C_{2,3}}$. Characteristic current waveforms can be seen on the ports 2 and 3, with ZCS turn-off before the end of the switching period. The current waveform of the port 1 , provides ZVS during the turn-on, while the turn-off is performed with a low current value defined by the magnetizing current.

Even if the loading at port 2 is modified (heavy load in Fig. 6, light load in Fig. 7), the current in port 3 in not affected and remains nearly the same. This decoupling is due to the low impedance of the tank in the vicinity of resonance frequency. In the case when port 2 is not loaded (Fig. 8), the resulting tank may be different, but the soft switching is still maintained.

\section{B. Operation in the Mode B}

In mode $\mathrm{B}$, two ports are providing power to the remaining port. Presented simulations show the case where port 1 and 3 are switched and are feeding port 2, where load is connected through a passive rectifier. Loading conditions on the port 2 are varied, as shown in Fig. 9 for the heavy load conditions and Fig. 10 for the light load conditions, respectively. Simulations demonstrate that the current in the rectifier of the port 2 maintains ZCS conditions and is softly commuted. The currents of the active ports 1 and 3 share the load provided to the port 2 while maintaining commutation conditions as in mode A. Note that both currents $I_{1}$ and $I_{3}$ are participating into $I_{m}$ and the power is shared with proportion in respect to the transformer turn ratio and the parameters of the resonant tank $\left(P_{1} / P_{3} \simeq 25\right)$. 

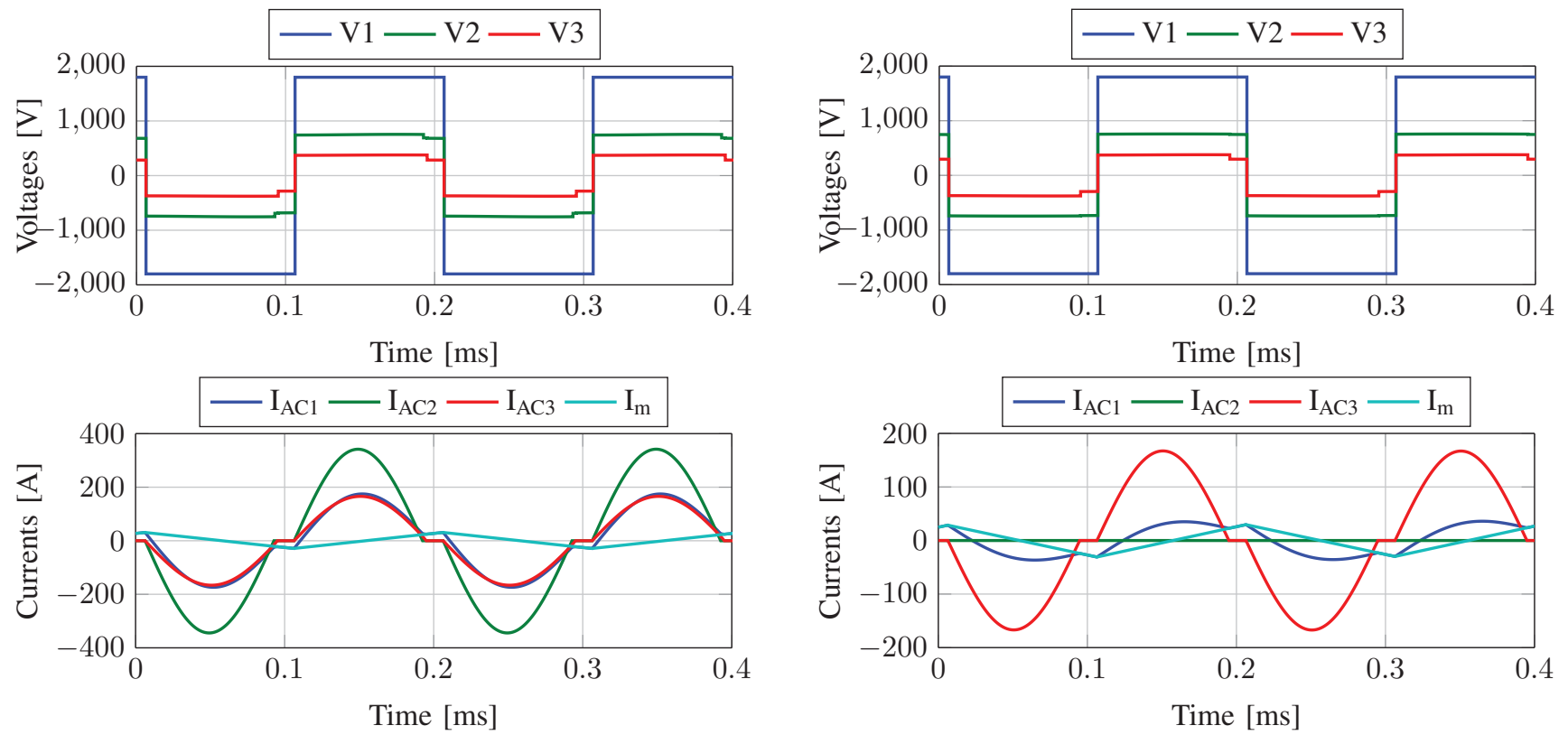

Fig. 6. Mode A: Source port: $1\left(P_{1} \simeq 215 k W\right)$; Sink ports: $2\left(P_{2} \simeq\right.$ $-172 k W)$ and $3\left(P_{3} \simeq-43 k W\right)$

Fig. 8. Mode A: Source port: $1\left(P_{1} \simeq 44 k W\right)$; Sink ports: $2\left(P_{2} \simeq 0 k W\right)$ and $3\left(P_{3} \simeq-44 k W\right)$
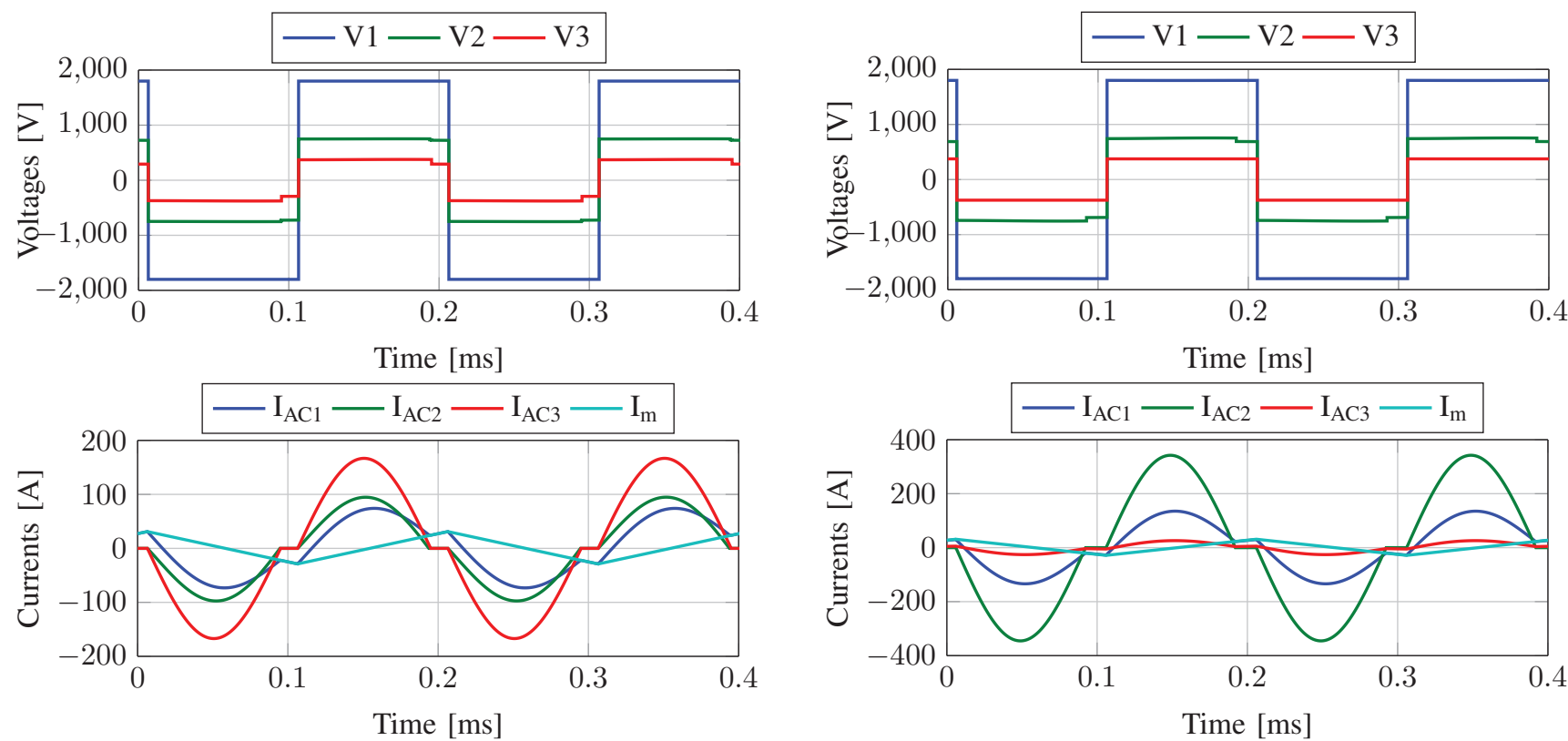

Fig. 7. Mode A: Source port: $1\left(P_{1} \simeq 92 k W\right)$; Sink ports: $2\left(P_{2} \simeq-48 k W\right)$ and $3\left(P_{3} \simeq-44 k W\right)$

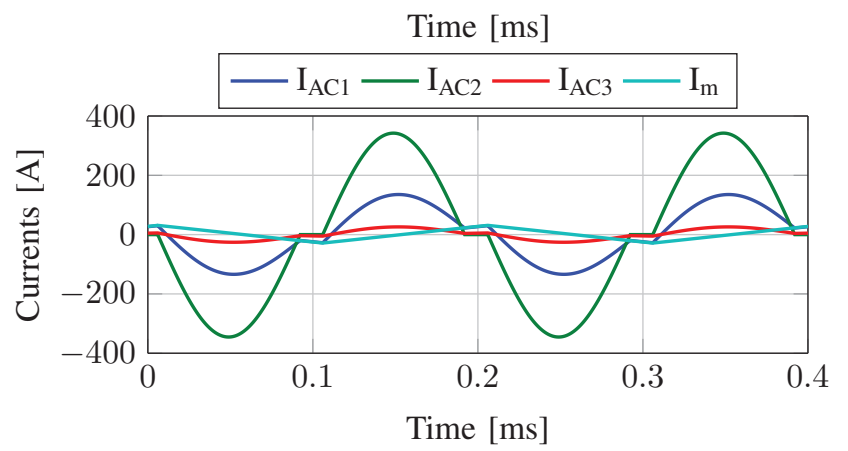

Fig. 9. Mode B: Source ports: $1\left(P_{1} \simeq 173 k W\right)$ and $3\left(P_{3} \simeq 7 k W\right)$; Sink port: $2\left(P_{2} \simeq-180 k W\right)$ 

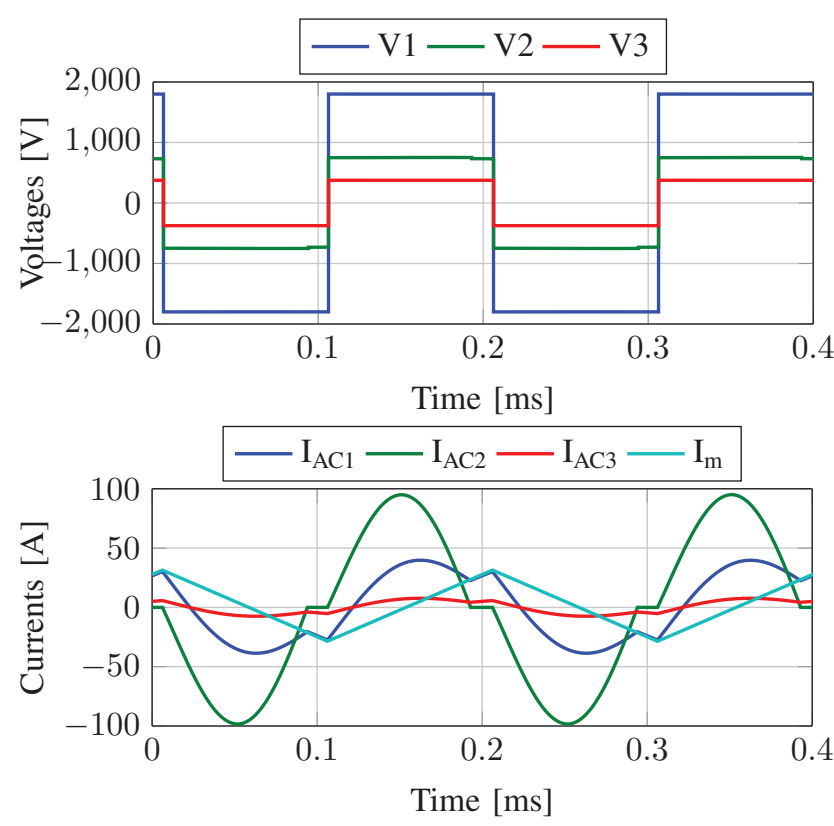

Fig. 10. Mode B: Source ports: $1\left(P_{1} \simeq 48 k W\right)$ and $3\left(P_{3} \simeq 2 k W\right)$; Sink port: $2\left(P_{2} \simeq-50 k W\right)$

\section{CONCLUSion}

The topology of a multi-port resonant DC-DC converter has been proposed in the paper, and is based on the use of a multi-winding MFT in combination with half-bridge converter stages and distributed resonant tank elements. Simple control scheme based on mode switching is proposed and criteria for the sizing of resonant tank components have been defined. The simulation results show that proposed topology is able to provide fully bi-directional power flow, while remaining in soft switching conditions over the wide range of operating conditions. Presence of switching actions define role of each port, and actual power sharing is influenced by the tank design. Additionally, if needed one port can be deactivated without disturbing the normal operation of remaining portion of the converter. Behavior of the converter resembles that of a DC transformer and to establish active power or voltage control additional power stages are required on some of the ports (depending on the application). Different rated voltages of ports imply use of semiconductor devices with different blocking voltages and while this is not elaborated in this paper, it requires careful considerations during the design.

\section{ACKNOWLEDGMENTS}

This research project is part of the National Research Programme "Energy Turnaround" (NRP 70) of the Swiss National Science Foundation (SNSF). Further information on the National Research Programme can be found at www.nrp70.ch.

\section{REFERENCES}

[1] J. Barton and D. Infield, "Energy storage and its use with intermittent renewable energy," Energy Conversion, IEEE Transactions on, vol. 19, no. 2, pp. 441-448, 2004.

[2] H. Tao, A. Kotsopoulos, J. Duarte, and M. Hendrix, "Family of multiport bidirectional dc-dc converters," Electric Power Applications, IEE Proceedings -, vol. 153, no. 3, pp. 451-458, 2006.

[3] H. Tao, A. Kotsopoulos, J. Duarte, and M. Hendrix, "Triple-half-bridge bidirectional converter controlled by phase shift and pwm," in Applied Power Electronics Conference and Exposition, 2006. APEC '06. TwentyFirst Annual IEEE, 2006, 7 pp.

[4] H. Tao, A. Kotsopoulos, J. Duarte, and M. Hendrix, "Transformer-coupled multiport zvs bidirectional dc/dc converter with wide input range," Power Electronics, IEEE Transactions on, vol. 23, no. 2, pp. 771-781, 2008.

[5] H. Tao, A. Kotsopoulos, J. Duarte, and M. Hendrix, "A soft-switched three-port bidirectional converter for fuel cell and supercapacitor applications," in Power Electronics Specialists Conference, 2005. PESC '05. IEEE 36th, 2005, pp. 2487-2493.

[6] Z. Pavlovic, J. A. Oliver, P. Alou, O. Garcia, and J. A. Cobos, "Bidirectional multiple port dc/dc transformer based on a series resonant converter," in Applied Power Electronics Conference and Exposition (APEC), 2013 Twenty-Eighth Annual IEEE, 2013, pp. 1075-1082.

[7] H. Krishnaswami and N. Mohan, "Constant switching frequency series resonant three-port bi-directional dc-dc converter," in Power Electronics Specialists Conference, 2008. PESC 2008. IEEE, 2008, pp. 1640-1645.

[8] I.-O. Lee and G.-W. Moon, "The k -q analysis for an llc series resonant converter," Power Electronics, IEEE Transactions on, vol. 29, no. 1, pp. 13-16, 2014.

[9] R.-L. Lin and C.-W. Lin, "Design criteria for resonant tank of llc dc-dc resonant converter," in IECON 2010. 36th Annual Conference on IEEE Industrial Electronics Society, 2010, pp. 427-432. 